\title{
EFFECT OF MULTIPLE GRAVITATIONAL LENSING ON THE ANISOTROPY OF THE COSMIC BACKGROUND RADIATION
}

\author{
T. FUKUSHIGE, J. MAKINO, AND T. EBISUZAKI \\ College of Arts and Sciences, University of Tokyo, \\ 3-8-1 Komaba, Meguro-ku, Tokyo 153, Japan
}

\begin{abstract}
We investigated smoothing of the cosmic background radiation (CBR) by multiple gravitational lensing. The CBR is gravitationally scattered by galaxies, clusters of galaxies, and superclusters during the travel from the last scattering surface. Although the effect of the gravitational lensing was thought to be unimportant, we found that the multiple gravitational lensing by clusters of galaxies or by superclusters can reduced by a large factor. This result is explained by the fact that the distance between two light rays grows exponentially though multiple gravitational lensing. If such structures were formed at $z=2-5$ and contain a large fraction of the mass of the universe $\left(\Omega_{\mathrm{s}}>0.5\right)$, then multiple gravitational lensing can reduce the temperature anisotropy of the CBR by $40-60 \%$, approximately up to a degree scale.
\end{abstract}

\section{Introduction}

The distance between rays grows exponentially, on average, until it reaches at the projected mean separation $d_{\mathrm{pr}}\left(=R N^{-1 / 2}\right)$ of lensing objects. The basic explanation of the exponential growth is given by Fukushige and Makino (in this proceeding, hereafter FM).

The possibility that the gravitational lensing change the anisotropy of the CBR has been discussed by many authors(e.g. Cole \& Efstathiou 1989, Sasaki 1989, Watanabe \& Tomita 1991). The light rays sampled in a radio telescope with a finite angular width come from a large angular region of the last scattering surface by gravitational lensing. This effect influences the anisotropy correlation function. However, most authors assumed that the effect of the multiple gravitational lensing could be expressed as the 


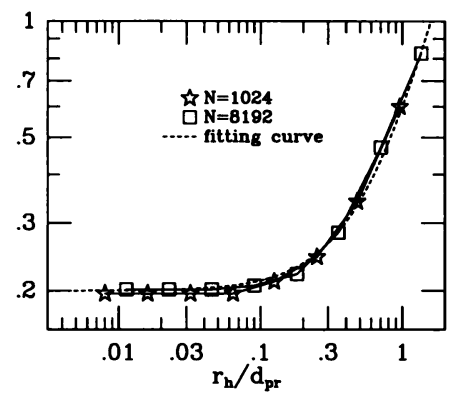

Figure 1. Non-dimensional $e$-folding time $(\tau)$ of the exponential growth plotted against half-mass radius $\left(r_{\mathrm{h}}\right)$ of lensing objects.

superposition of single scatterings. Thus their estimates did not take into account the exponential growth.

\section{Numerical Experiment}

The exponential growth does not occur if the universe is completely uniform. The scattering objects must be compact enough to be effective. We investigate the effect of the size of lensing object by 3D "ray tracing" simulations. The simulation is the same as that described in FM. The lensing object has the density profile of a King model $\left(W_{0}=5\right)$. In Figure 1 , the non-dimensional $e$-folding time of exponential growth $(\tau)$, scaled by $1 / \sqrt{G \rho}$, is plotted as a function of the half-mass radius of lensing objects $\left(r_{h}\right)$. The exponential growth is suppressed if $r_{\mathrm{h}}$ is larger than $d_{\mathrm{pr}}$.

We estimated the growth factor $\alpha_{\mathrm{LS}}$ of the angle between two photons that come from the last scattering surface by solving differential equations for the angle $\theta$ and the distance $w: c \theta=-d w / d t$ and $d w / d t=-c \theta_{0}-$ $w /(\tau / \sqrt{G \rho})$. The growth factor is estimated to be $\alpha_{\mathrm{LS}}=4-5$ if the superclusters were formed at $z=2$ and the universe consists only of the superclusters. The actual effect on the measured anisotropy depends on the power spectrum of the anisotropy, but it is likely to be significant. More detailed discussion is presented by Fukushige et al. $(1994,1995)$.

\section{References}

Cole, S., \& Efstathiou, G., 1989, MNRAS, 239, 195.

Fukushige, T., Makino, J., \& Ebisuzaki, T., 1994, ApJ, 436, L107.

Fukushige, T., Makino, J., Nishimura, O., \& Ebisuzaki, T., 1995, PASJ, in press.

Sasaki, M., 1989, MNRAS, 240, 415.

Watanabe, K., \& Tomita, K., 1991, ApJ, 370, 481. 